\title{
Formation and stability of new palm-based nanoemulsions
}

\begin{abstract}
Nanoemulsions as vehicle have found many applications in cosmetic and medicinal industry attributable to its enormously low droplets size, high droplets volume and exceptional stability. Nanoemulsions are made of very fine oil-in-water dispersion, having oil droplets ranging from 80 to $200 \mathrm{~nm}$. In this work, the behaviour of palm oil esters (POEs) (Gunawan et al., 2004 E.R. Gunawan, M. Basri, M.B.A. Rahman, A.B. Salleh and R.N.Z. Rahman, Lipased-catalyzed synthesis of palm-based wax esters, J. Oleo Sci. 53(10). (2004), pp. 471477.[Gunawan et al., 2004], [Mat Radzi et al., 2006] and [Basri et al., 2007]) as the lipophilic phase was studied with nonionic surfactants and water. The palm-based nanoemulsions were prepared using high-energy emulsification to supply the emulsion system with sufficient amount of energy as to form the dispersed surfactant-encapsulated nanodroplets. The effects of amount of surfactant and different pressures, cycles, stirring rate and duration were studied to produce the nanodroplets. Stability of the palm-based nanoemulsions was also investigated with regards to their Ostwald ripening rate. The results showed that the type and composition of surfactant were important for the formation and stability of the nanoemulsions. Lipophilic surfactant, SPAN® 20 gave the smallest droplets size range $(78-120 \pm 2 \mathrm{~nm})$ as compared to hydrophilic surfactant, Tween ${ }^{\circledR} 60(105-180 \pm \mathrm{nm})$. Introducing high pressure and cycle reduced the size tremendously after the pre-mixing. Stirring rate and duration also affected the nanodroplets formation. Increasing processing temperature led to the deformation of the nanodroplets which is the key finding in the searching of emulsification route that produce the most stable system. The investigation on the formation and stability of palm-based nanoemulsions is very important in the preparation for poorly water-soluble lipophilic actives/drugs for cosmetic and medicinal formulations. Moreover, its water-like fluidic rheological behavior is excellent as a vehicle for topical cosmetics and pharmaceuticals.
\end{abstract}

Keyword: nanoemulsions, palm-oil, lipase-catalyzed synthesis 